\title{
Magnetic Compression Duct-to-duct Anastomosis for Biliary Obstruction in a Patient with Living Donor Liver Transplantation
}

\author{
Takao Itoi ${ }^{\star}$, Eigoro Yamanouchi ${ }^{\dagger}$, Nobuhito Ikeuchi $^{\star}, K^{\prime}$ azuhiko Kasuya ${ }^{\ddagger}$, Hitoshi Iwamoto ${ }^{\S}$, and Akihiko \\ Tsuchida \\ *Departments of Gastroenterology and Hepatology, Tokyo Medical University, Tokyo, ${ }^{\dagger}$ Department of Radiology, Yokohama Seibu Hospital, \\ St. Marianna University, Yokohama, ${ }^{\neq}$Third Department of Surgery, Tokyo Medical University, Tokyo, and ${ }^{\xi}$ Fifth Department of Surgery, \\ Hachioji Medical Center, Tokyo Medical University, Tokyo, Japan
}

\begin{abstract}
Magnetic compression anastomosis (MCA) is a minimally invasive method of performing choledochocholedochostomy without surgery in patients with biliary stricture or obstruction. We describe a successful case involving magnetic compression duct-to-duct biliary reconstruction in right-lobe living donor liver transplantation (RL-LDLT). Endoscopically, a samarium-cobalt (SmCo) rare-earth magnet was placed at the superior site of obstruction via the percutaneous transhepatic biliary drainage route, and another Sm-Co magnet was also placed at the inferior site of obstruction with the aid of an endoscope. MCA techniques enabled complete anastomosis without procedure-related complications. In conclusion, the MCA technique is a revolutionary method of performing choledochocholedochostomy in patients with biliary obstruction after LDLT. (Gut Liver 2010;4(Suppl. 1):S96-98)
\end{abstract}

Key Words: Magnetic compression anastomosis; Biliary stricture; Biliary obstruction; Choledochocholedochostomy; Choledochoenterostomy

\section{INTRODUCTION}

The magnetic compression duct-to-duct biliary reconstruction has been developed as minimum invasive choledochocholedochostomy without surgery. ${ }^{1-7}$ It can create a non-surgical, sutureless enteric anastomosis is conjunction with interventional radiologic technique by using two magnets showing strong power. Herein, we described the successful case in which magnetic compression duct-to- duct biliary reconstruction in right-lobe living donor liver transplantation (RL-LDLT).

\section{CASE REPORT}

A 60-year-old man presented with obstructive jaundice. He had undergone RL-LDLT 18 months ago. Laboratory liver function tests revealed a cholestatic pattern of liver dysfunction, with a bilirubin of $3.1 \mathrm{mg} / \mathrm{dL}$. Computed tomography showed a dilatation of right posterior intrahepatic bile duct (Fig. 1A). Then, we attempted transpapillary biliary drainage but right posterior intrahepatic bile duct was invisible by endoscopic retrograde cholangiopancreatography (ERCP) (Fig. 1B). Furthermore, an antegrade guidewire passing through the percutaneous transhepatic biliary drainage (PTBD) route was impossible (Fig. 1C). Therefore, we conducted magnetic compression anastomosis (MCA) as we previously described ${ }^{2,3}$ after obtaining written informed consent. In brief, the MCA technique was applied in the ERCP procedure room. We prepare two magnets, parent and daughter magnet. First, the 14-Fr PTBD catheter was replaced. A cylindrical samarium-cobalt $(\mathrm{Sm}-\mathrm{Co})$ rare-earth magnet (daughter magnet), $4 \mathrm{~mm}$ in diameter, attached to a $30 \mathrm{~cm}$ nylon wire, was placed inside a sheath. Then, endoscopic sphincterotomy (EST) and the papillary balloon dilation (Haricane, maximum diameter: $10 \mathrm{~mm}$; Boston Scientific Japan, Tokyo, Japan) are performed to easily advance the magnet into bile duct. Once the endoscope was withdrawn, a Sm-Co rare-earth magnet (parent magnet), 4 to $5 \mathrm{~mm}$ in

\footnotetext{
Correspondence to: Takao Itoi

Department of Gastroenterology and Hepatology, Tokyo Medical University, 6-7-1 Nishishinjuku, Shinjuku-ku, Tokyo 160-0023, Japan

Tel: +81-3-3342-6111, Fax: +81-3-5381-6654, E-mail: itoi@tokyo-med.ac.jp DOI: $10.5009 /$ gnl.2010.4.S1.S96
} 

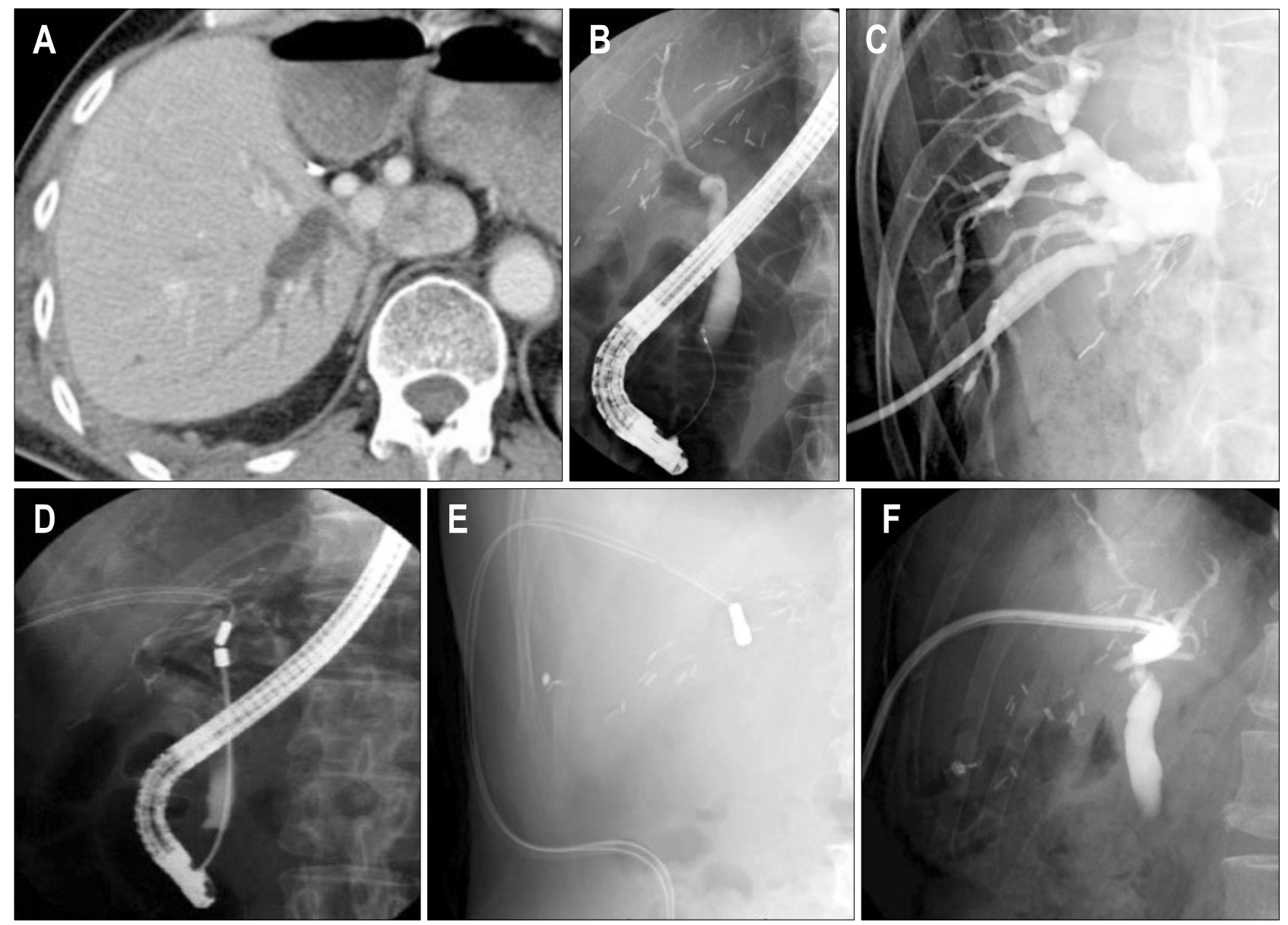

Fig. 1. (A) Computed tomography showed dilation of the right-posterior intrahepatic bile duct. (B) The right-posterior intrahepatic bile duct was not visible in endoscopic retrograde cholangiography. (C) Percutaneous transhepatic biliary drainage cholangiogram revealed a complete obstruction between the common bile duct and the right-posterior intrahepatic bile duct. (D) The magnet was positioned at the site of the obstruction in the common bile duct via the papilla of Vater. (E) Both magnets were advanced to immediately prior to the biliary obstruction, and it was confirmed that the two magnets attracted each other. (F) Recanalization was achieved at 9 days after the procedure without any adverse event.

diameter with a nylon handle attached, was clasped with standard polypectomy snare forceps (SD-5U-1, 6U-1; Olympus Medical Systems, Tokyo, Japan) while the endoscope is re-inserted up to the duodenum, and the magnet is positioned in the common bile duct via the papilla of Vater (Fig. 1D). Both magnets were advanced to immediately prior to the biliary obstruction and it was confirmed that the two magnets attract each other magnetically (Fig. 1E). Nine days after the procedure, recanalization could be achieved without any adverse event (Fig. 1F). Finally, a 16-Fr PTBD catheter was placed across the created fistula. Six months after creation of fistula, the PTBD tube was removed. Over a 2 months period up to the present, neither recurrence of obstruction nor other complications have been recognized.

\section{DISCUSSION}

Nowadays, LDLT is one of acceptable surgery in patients with serious liver diseases. In LDLT patients, anastomotic stricture, duct-to-duct or duct-to-GI tract, is the most common and problematic adverse events up to $30 \%{ }^{8,9}$ There are several interventions including re-operation, interventional radiology, and endoscopic therapy to resolve the tough stricture.

In the present case, successful MCA was performed without any adverse events. Basically, accomplishment of MCA depends on the distance between two magnets and the power of magnet. In our personal opinion, magnetic duct-to-duct anastomosis after LDLT is easily performed because the length of obstruction is relatively short like this case. In our experimentation, two magnets (each has 
3,000 gauss power) were attracted each other less than 20-mm (data not shown). Therefore, we have to perform this procedure after confirming the length of obstruction. In addition, we need to check the intervening blood vessel between two magnets to avoid serious adverse events.

Duct-to-duct anastomosis using MCA is technically difficult for accomplishment of MCA because positioning a parent magnet in the common bile duct via the papilla of Vater toward the obstruction site is cumbersome or occasionally impossible because we developed this procedure for ERCP without endoscopically dedicated magnets. Furthermore, this procedure limits because PTBD is necessary. Nevertheless, duct-to-duct was selected for the following reasons: i) nature bile flow may be important for patients, ii) creation of unnecessary fistula may be able to avoid early or late complications like a ruptured blood vessels. So far, in the process of anastomosis, there have been no report on the ruptured blood vessels or other major complications, but as we described, we should take care of intervening blood vessels between two magnets.

In conclusion, although further accumulation of clinical cases and improvement of the dedicated magnet for duct-to-duct during ERCP are necessary, we believe that this method will be promising in re-canalization in patients with biliary obstruction following living donor liver transplantation.

\section{ACKNOWLEDGEMENTS}

The authors have no commercial associations that might pose a conflict of interest in relation to this article.

\section{REFERENCES}

1. Takao S, Matsuo Y, Shinchi H, et al. Magnetic compression anastomosis for benign obstruction of the common bile duct. Endoscopy 2001;33:988-990.

2. Mimuro A, Tsuchida A, Yamanouchi E, et al. A novel technique of magnetic compression anastomosis for severe biliary stenosis. Gastrointest Endosc 2003;58:283-287.

3. Itoi $\mathrm{T}$, Yamanouchi $\mathrm{E}$, Ikeda $\mathrm{T}$, et al. Magnetic compression anastomosis: a novel technique for canalization of severe hilar bile duct strictures. Endoscopy 2005;37: 1248-1251.

4. Akita H, Hikita H, Yamanouchi E, et al. Use of a metallic-wall stent in the magnet compression anastomosis technique for bile duct obstruction after liver transplantation. Liver Transpl 2008;14:118-120.

5. Muraoka N, Uematsu H, Yamanouchi E, et al. Yamanouchi magnetic compression anastomosis for bilioenteric anastomotic stricture after living-donor liver transplantation. J Vasc Interv Radiol 2005;16:1263-1267.

6. Okajima H, Kotera A, Takeichi $\mathrm{T}$, et al. Magnet compression anastomosis for bile duct stenosis after ductto-duct biliary reconstruction in living donor liver transplantation. Liver Transpl 2005;11:473-475.

7. Matsuno N, Uchiyama M, Nakamura Y, et al. A nonsuture anastomosis using magnetic compression for biliary stricture after living donor liver transplantation. Hepatogastroenterology 2009;56:47-49.

8. Kawachi S, Shimazu M, Wakabayashi G, et al. Biliary complications in adult living donor liver transplantation with duct-to-duct hepaticocholedochostomy or Roux-en-Y hepaticojejunostomy biliary reconstruction. Surgery 2002;132: 48-56.

9. Venu M, Brown RD, Lepe $\mathrm{R}$, et al. Laboratory diagnosis and nonoperative management of biliary complications in living donor liver transplant patients. J Clin Gastroenterol 2007;41:501-506. 